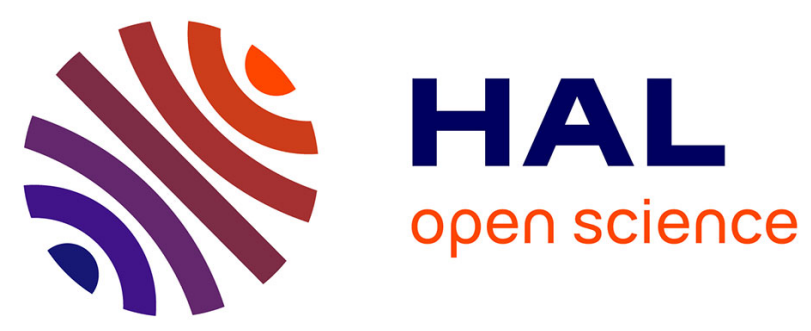

\title{
Prediction model adaptation thanks to control chart monitoring application to pollutants prediction
}

Philippe Thomas, William Derigent, Marie-Christine Suhner

\section{To cite this version:}

Philippe Thomas, William Derigent, Marie-Christine Suhner. Prediction model adaptation thanks to control chart monitoring application to pollutants prediction. 6th International Joint Conference on Computational Intelligence, IJCCI'14, Oct 2014, Rome, Italy. hal-01086851

\section{HAL Id: hal-01086851 https://hal.science/hal-01086851}

Submitted on 25 Nov 2014

HAL is a multi-disciplinary open access archive for the deposit and dissemination of scientific research documents, whether they are published or not. The documents may come from teaching and research institutions in France or abroad, or from public or private research centers.
L'archive ouverte pluridisciplinaire $\mathbf{H A L}$, est destinée au dépôt et à la diffusion de documents scientifiques de niveau recherche, publiés ou non, émanant des établissements d'enseignement et de recherche français ou étrangers, des laboratoires publics ou privés. 


\title{
Prediction model adaptation thanks to control chart monitoring. Application to pollutants prediction
}

\author{
Philippe THOMAS ${ }^{1,2}$, William DERIGENT $^{1,2}$ and Marie-Christine SUHNER ${ }^{1,2}$ \\ ${ }^{1}$ Université de Lorraine, CRAN, UMR 7039, Campus Sciences, BP 70239, 54506 Vandouvre-lès-Nancy cedex, France \\ ${ }^{2}$ CNRS, CRAN, UMR7039, France \\ \{philippe.thomas, william.derigent, marie-christine.suhner\}@univ-lorraine.fr
}

\begin{abstract}
Keywords: indoor air quality, neural networks, relearning, control charts
Abstract: Indoor air quality is a major determinant of personal exposure to pollutants in today's world since people spend much of their time in numerous different indoor environments. The Anaximen company develops a smart and connected object named Alima, which can measure every minute several physical parameters: temperature, humidity, concentrations of $\mathrm{COV}, \mathrm{CO}_{2}$, formaldehyde and particulate matter (pm). Beyond the measurement aspect, Alima presents some data analysis feature named 'predictive analytics', whose primary aim is to predict the evolution of indoor pollutants in time. In this article, the neural network (NN) model, embedded in this object and designed for pollutant prediction, is presented. In addition with this NN model, this article also details an approach where batch learning is performed periodically when a too important drift between the model and the system is detected. This approach is based on control charts.
\end{abstract}

\section{INTRODUCTION}

Air pollution is now identified as a major international issue. However, in people's mind, it always refers to the quality of outdoor air, whereas the predominant environment in this regard is the residence. Indeed, indoor air quality is a major determinant of personal exposure to pollutants in today's world since people spend much of their time in numerous different indoor environments (Walsh et al. 1987).

During the last two decades there has been increasing concern within the scientific community over the effects of indoor air quality on health. Changes in building design devised to improve energy efficiency have meant that modern homes and offices are frequently more airtight than older structures. Furthermore, advances in construction technology have caused a much greater use of synthetic building materials, which provide indoor pollution (Jones 1999).

The known health impacts and corresponding pollutants are numerous. Table 1 is an excerpt taken from (Spengler and Sexton, 1983) to illustrate some of the major indoor pollutants. The sources of pollution can be located indoor (building material, furniture, stoves...) or outdoor (air coming through an opened window or via the ventilation system).

Table 1. Pollutants and sources

\begin{tabular}{|c|c|}
\hline Pollutant & Major emission sources \\
\hline Allergens & House dust, domestic animals, \\
\hline Asbestos & Fire retardant materials, insulation \\
\hline Carbon dioxide & Metabolic activity, combustion activities, \\
\hline Carbon monoxide & Fuel burning, boilers, stoves, gas... \\
\hline Formaldehyde & Particleboard, insulation, furnishings \\
\hline Micro-organisms & People, animals, plants, air conditioning \\
\hline Nitrogen dioxide & Outdoor air, fuel burning, motor vehicles \\
\hline Organic substances & Adhesives, solvents, building materials, \\
\hline Ozone & Photochemical reactions \\
\hline Particles & Tobacco smoke, combustion products... \\
\hline $\begin{array}{c}\text { Polycyclic aromatic } \\
\text { hydrocarbons }\end{array}$ & Fuel combustion, tobacco smoke \\
\hline Pollens & Outdoor air, trees, grass, weeds, plants \\
\hline Radon & $\begin{array}{l}\text { Soil, building construction materials } \\
\text { (concrete, stone) }\end{array}$ \\
\hline Fungal spores & Soil, plants, foodstuffs, internal surfaces \\
\hline Sulfur dioxide & Outdoor air, fuel combustion \\
\hline
\end{tabular}

Symptoms and consequences of exposure to a pollutant can vary depending on the pollutant type and concentration. For example, the carbon dioxide (whose indoor concentrations can vary from 700 to 
$3000 \mathrm{ppm}$ ) is a simple suffocating gas and can also act as a respiratory irritant (Maroni et al. 1995), whereas the exposition to a formaldehyde concentration of $100 \mathrm{ppm}$ can cause death.

It thus explains why indoor air quality recently receives much public attention, and people are now eager to measure in their own homes the quality of their indoor air. To answer to this growing need, The Anaximen Company develops a smart and connected object named Alima (Alima, 2013).

In fact, pollutant levels are constantly changing, depending on the tenants' activities. Alima can measure every minute several physical parameters: temperature, humidity, concentrations of $\mathrm{COV}, \mathrm{CO}_{2}$, formaldehyde and particulate matter $(\mathrm{pm})$. Data are stored on the object or can be sent to a distant database, and are available for the user online via phone apps or websites. Beyond the measurement aspect, the society currently plans to embed in Alima some data analysis feature named 'predictive analytics', whose primary aim is to predict the evolution of indoor pollutants in time. Anaximen and the CRAN laboratory are associated to develop this leading-edge feature.

In this article, a neural network (NN) model designed for pollutant prediction is presented. However, a drift can appear between the NN model and the system modelled (due to, for example, a modification of the occupant behavior), and forces the $\mathrm{NN}$ to do a relearning phase. But this relearning phase is time and resource consuming and should be done sparingly. So, in addition with the NN model for pollutant detection, this article also details an approach where batch learning is performed periodically when a too important drift between the model and the system is detected. This approach is based on control charts.

Section 2 presents a short state-of-the-art on neural network modelling, section 3 details the approach used to control the drift and perform the batch learning while section 4 presents the industrial application. Section 5 first exposes the results obtained by the NN model for pollutant prediction (without any relearning phase), and then describes the results obtained when using the drift detection algorithm.

\section{NEURAL NETWORK MODEL}

Artificial neural network models have been successfully applied to solve many different problems, including dynamic systems identification, patterns classification, adaptive control, functions approximation and so on.
Among these artificial neural network models, the multilayer perceptron (MLP) is, by far, the most popular architecture due to its structural flexibility, good representational capabilities, and the availability of a large number of training algorithms (Han and Qiao 2013). This model is used for both classification and regression tasks.

Works of Cybenko (1989) and Funahashi (1989) have proved that a MLP with only one hidden layer using a sigmoïdal activation function and an output layer can approximate all non-linear functions with the wanted accuracy. Its structure is given by:

$$
z=g_{2}\left(\sum_{i=1}^{n_{1}} w_{i}^{2} \cdot g_{1}\left(\sum_{h=1}^{n_{0}} w_{i h}^{1} \cdot x_{h}^{0}+b_{i}^{1}\right)+b\right)
$$

Where:

- $\quad x_{h}^{0}$ are the $n_{0}$ inputs of the neural network,

- $\quad w_{i h}^{1}$ are the weights connecting the input layer to the hidden layer,

- $\quad b_{i}^{1}$ are the biases of the hidden neurons,

- $g_{1}($.$) is the activation function of the hidden$ neurons (namely the hyperbolic tangent),

- $w_{i}^{2}$ are the weights connecting the hidden neurons to the output one,

- $\quad b$ is the bias of the output neuron,

- $g_{2}($.$) is the activation function of the output$ neuron and,

- $\quad z$ is the network output.

Because the problem is a regression problem, $g_{2}($.$) is$ chosen linear.

Three steps must be performed in order to design the neural model: initialization, learning and pruning.

The first one is the determination of the initial set of weights and biases. This step is important because learning algorithm performs a local search of the minimum. So, in order to avoid local minimum trapping, different initial sets must be constructed which allow beginning to learn in different zones of the criteria domain. Different initialization algorithms have been proposed in the literature (Thomas and Bloch 1997). The initialization algorithm used in this paper is the one proposed by Nguyen and Widrow (1990) which allows associating a random initialization of weights and biases to an optimal placement in input and output spaces.

The second step is performed by the learning algorithm which must fit the network output with the data. In industrial applications, data are noisy and corrupted with many outliers. In order to limit the 
impact of outliers on the results, a robust LevenbergMarquardt algorithm is used (Thomas et al. 1999). Levenberg-Marquard algorithm allows associating the speed of the Hessian methods to the stability of the gradient methods. This is performed by adding a parameter multiplied by the identity matrix in order to permit the inversion of the Hessian matrix even if it is singular. The tuning of this parameter during the learning allows the Levenberg-Marquard algorithm to work as a gradient descent algorithm when this parameter is large and as a Gauss-Newton algorithm when this parameter is small. The use of a robust criterion allows to avoid the influence of outliers and provides a regularization effect in order to prevent overfitting. An important issue in neural network design is the determination of its structure. To determine it, two approaches can be used. The first one is constructive, where the hidden neurons are added one after the other (Ma and Khorasani 2004). The second approach exploits a structure with too many initial hidden neurons, and then prunes the least significant ones (Setiono and Leow 2000, Engelbrecht 2001). We focus on the pruning approach that allows a simultaneous selection of the input neurons and the number of hidden neurons. The pruning phase is performed in two steps. First, the Engelbrecht algorithm is used which allows to quickly simplify the structure and second, the Setiono and Leow algorithm is used which is slower but also more efficient (Thomas et al. 2013).

\section{ONLINE ADAPTATION OF THE MODEL}

\subsection{Generalities}

Ideally, the data collected during the experimentation phase should describe all the states of the system to model. However, it is sometimes not feasible due to the high number of potential situations the system could encounter. Indeed, in our case data collected are different depending on the seasons, the yearly weather, changes in user's habits and so on. Technically, it would thus be highly difficult to obtain an exhaustive data set. As a result, our approach consists in two phases: first, a learning phase is achieved based on a data set obtained via a relatively short experimentation phase (in our case, 1 month, see section 4.1) to construct a first "specific" NN model. Then, a relearning is launched if and only if a significant difference (called "drift") between the system behavior and its corresponding NN model is detected.

In many case, a drift may appear between the model constructed and the system studied. This drift may be due to two main reasons. The first one concerns the evolution of input parameters. With a learning approach, the obtained model is valid only on the learned domain. The model is able to provide a valid solution only in this concerned domain.

The second reason concerns the uncontrolled modification of the machine or environment behavior. Indeed, A change of a parameter (voluntarily or not, measured or not) which is not an input of the model, can affect the behavior of the machine. In this case, this parameter should be part of the model inputs but, as it was considered constant for the duration of the learning step, it was not retained as such. Due to this change, which may even be unknown to operators and users, the model will therefore provide results out of step with reality.

To take into account these problems, a relearning on new data is needed. There are two practical ways to implement learning in neural networks: batch training and on-line training. Whenever a new data is received, batch learning uses this new data together with the past data to perform a retraining. But this approach is time consuming. The on-line approach uses only new data to adapt the model. However, this approach suffers from slow training error convergence as a large number of training data may be required (Liang et al. 2006). Moreover, different works have shown that on-line training strategy does not converge to the optimal weights (Heskes and Wiegerinck 1996, Nakama 2009).

We thus propose here another approach where a batch learning is performed periodically when a drift between the model and the system occurs, in order to synchronize the model with the reality.

Because this synchronization is time consuming, the synchronization frequency must be optimized. Rather than consider a resynchronization frequency in response to events (arrival of new information from one of the connected devices, solicitation by an operator...) or a periodically one (every hour, week...), it is better to rely on statistical findings. Among the 7 basic tools for quality control, control charts, also known as Shewhart charts or processbehavior charts (Shewhart 1931), are interesting Statistical Process Control (SPC) tools useful for our proposed system. 


\subsection{Control charts}

Control charts are particularly relevant to the dynamic quality control with the use of time-series (Tague 2004). They can determine statistically if a variation is no longer under control. Indeed, it is known that even when a process is under control there is approximately a $0.27 \%$ probability of a point exceeding a $3 \sigma$ control limit (Pareto). These few isolated points should not trigger synchronization. But the detection of too many points above this limit may underlines the presence of a special cause, even if it is not yet known.

The Combination of a neural network with the control charts can therefore inherit from the robustness of the statistical analysis and the adaptability of the neural network. Du et al. (2012) work on the inverse combination of both tools with a recognition algorithm of control charts using neural networks to get alerts in case of quality problems and to provide clues in defining causes.

In our case, when a set of $n$ new data is collected, a performance indicator is calculated and compared to two bounds determined on the initial validation data set. If this indicator is still between the two bounds, the model is always suitable. If the new value of the indicator is outside the bounds, a relearning phase is needed on these new collected data. Figure 1 shows an example of control chart to monitor the accuracy of the model. It presents the evolution of the considered indicator compared to the upper (UCL) and lower bounds (LCL) in function of samples of size $n$. In this example, the two first samples present acceptable results and the model accuracy is sufficient. For the third sample, the indicator is outside the bounds and a relearning on the $n$ last data is needed to drive the indicator inside the bounds for the next samples. If no relearning occurs, the drift between model and reality stays and may grow.

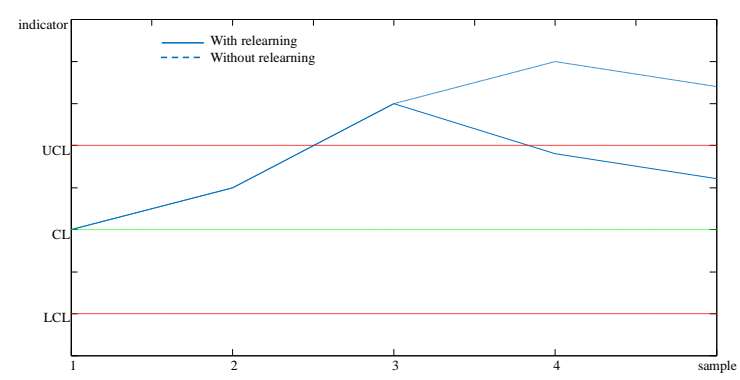

Figure 1: Example of control chart used for monitoring accuracy of the model.

\section{3 control bounds}

Our control charts (s charts) aim to determine if a process characteristic is stable. The center line (CL) is given by the standard deviation of the considered characteristics. The Upper Control Limit (UCL) and the Lower Control Limit (LCL) are calculated for representing $99.8 \%$ of data (NIST/SEMATECH 2012). These limits are given by:

$$
\left\{\begin{array}{l}
U C L=\bar{s}+3 \frac{\bar{s}}{c_{4}} \sqrt{1-c_{4}^{2}} \\
C L=\bar{s} \\
L C L=\bar{s}-3 \frac{\bar{s}}{c_{4}} \sqrt{1-c_{4}^{2}}
\end{array}\right.
$$

where:

- $\bar{s}$ stands for the center line (CL) and correspond to the estimated standard deviation of the characteristic monitored,

- $\quad n$ is the size of the sample,

- $\quad c_{4}$ is a factor allowing to find an unbiased estimator of the standard deviation $\sigma$.

$$
\bar{s}=c_{4} \cdot \sigma
$$

With $c_{4}$ given by:

$$
c_{4}=\sqrt{\frac{2}{n-1}} \frac{\left(\frac{n}{2}-1\right) !}{\left(\frac{n-1}{2}-1\right) !}
$$

where the non-integer factorial is given by:

$$
\left(\frac{n}{2}\right) !=\left(\frac{n}{2}\right) \cdot\left(\frac{n}{2}-1\right) \cdot\left(\frac{n}{2}-2\right) \cdots\left(\frac{1}{2}\right) \cdot \sqrt{\pi}
$$

In the considered case, the monitored characteristic is the error performed by the network, and so, the parameter to monitor is the sample standard deviation given by:

$$
s=\sqrt{\frac{\sum_{i=1}^{n}\left(\varepsilon_{i}-\bar{\varepsilon}\right)^{2}}{n-1}}
$$

where $\varepsilon_{i}$ stands for the error performed on data $i$ and $\bar{\varepsilon}$ stands for the mean of the error.

The value of the estimated standard deviation $\bar{s}$ is obtained on the validation data set used to validate the initial model. 


\section{INDUSTRIAL APPLICATION}

\subsection{Description of the case study}

The experimentation site is a single storey dwelling whose floor plan is shown figure 2 .

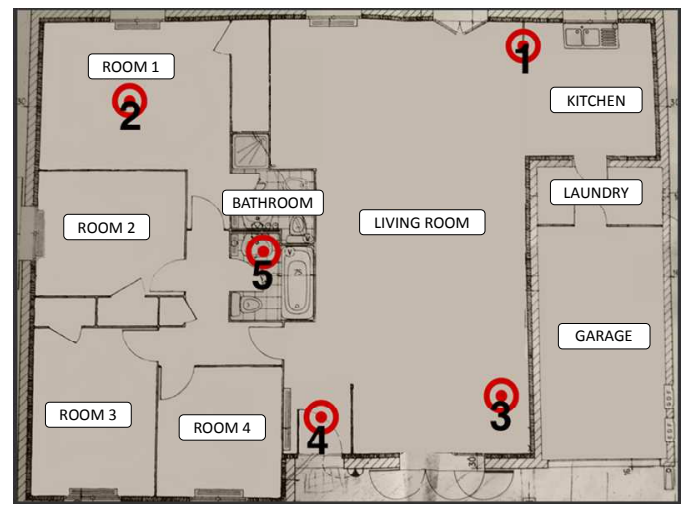

Figure 2: Implantation of the 5 Alima in a house.

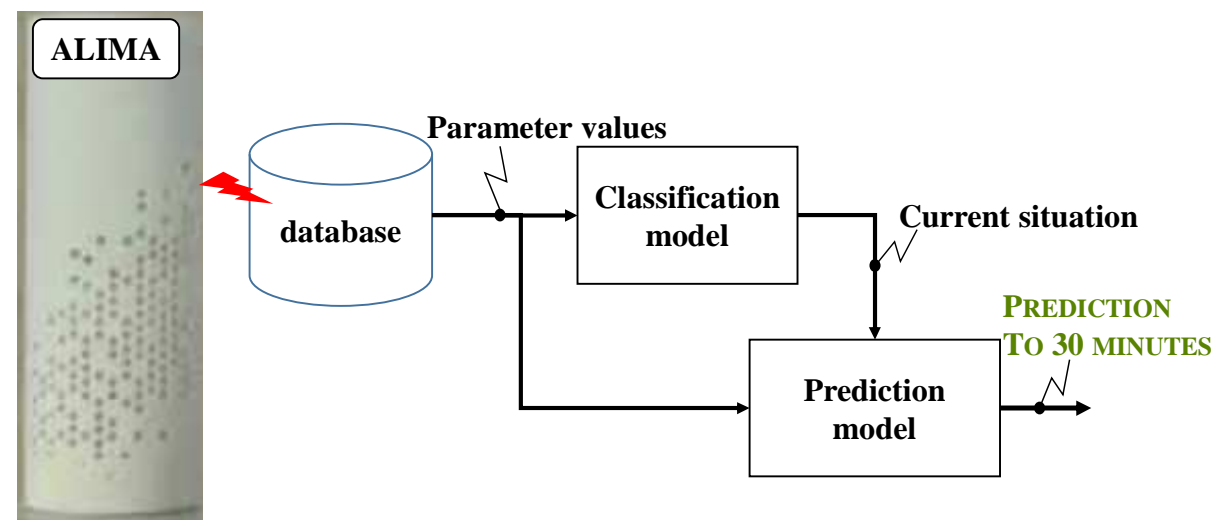

Figure 3: Pollutant prediction principle.

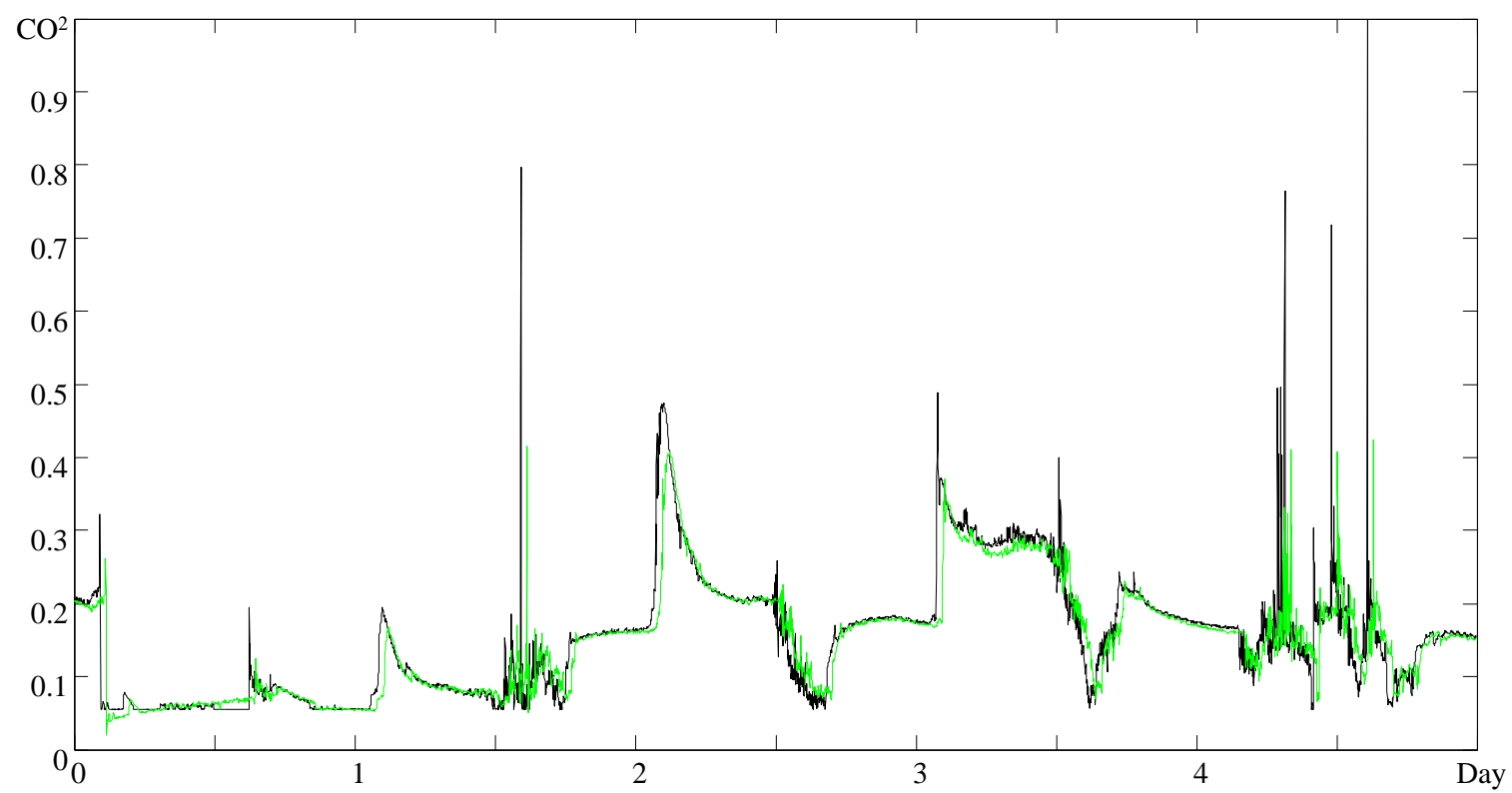

Figure 4: prediction of $\mathrm{C}^{2}$. 
Red points indicate the different locations of the 5 Alimas installed in the house. The experimentation ran during a period of 1 month during summer 2013, and Alimas were recording each minute the values of their different sensors. The data set is divided in two subsets (learning and validation data set) each corresponding to 15 days of collected data. Only data collected by Alima1 are used for the learning and validation.

\subsection{Considered problem}

We focus here on the problem of pollutant evolution prediction. As explain previously, each Alima collects, each minute, the values of five parameters (temperature, humidity, $\mathrm{CO}^{2}, \mathrm{COV}$ and particulate matter (pm)). The goal is to predict 30 minutes ahead the level of each pollutant in function of the actual and past level of these pollutants. In order to improve the performance of the model, different situations (cooking, sleeping...) are detected by using a classification model. The output of this classification model is used as an input of the prediction model.

The figure 3 presents the principle of the prediction of pollutants. The database collects data from the Alima. These data are use in a first step in order to detect the current situation (cooking, sleeping...) which may have an impact on the pollutant evolution. The design of the classification model has already been submitted to the MOSIM2014 conference. This article is thus entirely focused on the prediction model. This model takes as inputs the 8 actual outputs of the classification model and the 5 past values $(t, t-1, t-2, t-3, t-4)$ of the parameters collected by Alima. Three different models are built for each pollutant $\left(\mathrm{CO}^{2}, \mathrm{COV}, \mathrm{pm}\right)$. The output of the considered model is the value of the considered pollutant 30 minutes later $(t+30)$. In order to avoid the local minimum trapping, the learning is performed on 20 different initial parameters sets. The data set is divided in two subsets (learning and validation data set) each corresponding to 15 days of collected data. Only data collected by Alima1 are used for the learning and validation. A pruning algorithm is used in order to avoid the overfitting problem. At last, the best resulting model is selected.

\section{RESULTS}

\subsection{Results obtained on the validation data set}

In a first step, the results obtained on the validation data set are presented. Figure 4 presents the prediction of $\mathrm{CO}^{2}$ (in green) to compare to the real values collected (in black). This figure shows that the evolution of $\mathrm{CO} 2$ level may be predicted with a good accuracy even if the amplitude of the larger variation can't be predicted.

The figure 5 presents the prediction error for the $\mathrm{CO}^{2}$ pollutant (for the first 5 days of the validation data set). This figure shows that the model is able to predict the smooth evolution but it is not able to find the total amplitude of greatest variations. For these two figures, the data are normalized due to confidential needing. However, the model works with the true range of variation.

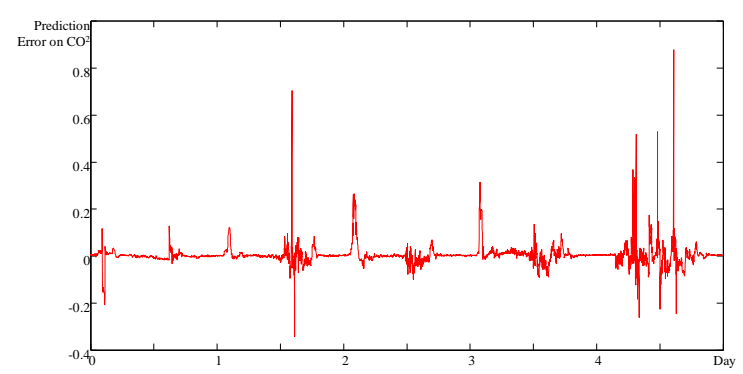

Figure 5: prediction error of $\mathrm{C}^{2}$.

The results obtained for the COV pollutant is quite similar and are not presented here.

Figure 6 and 7 present the same results obtained for pm pollutants.

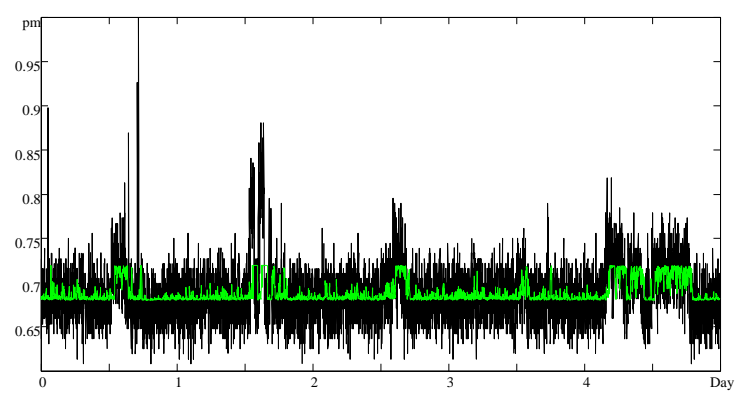

Figure 6: prediction of pm.

These two figures shows that pm is corrupted with an important noise. The events to detect have an amplitude of the same order to the noise variance. However, the model is able to predict the evolution of the pm pollutant with a good accuracy.

\subsection{Adaptation of the models}

The main goal of this model is to be suitable even if the conditions change (move in the house, or change 
of house ...) and the model must to be portable from one Alima to another. In order to do that, the model must be adaptable on-line. To do that, we propose to detect if the model varies from the reality, and to perform a relearning only if needed.

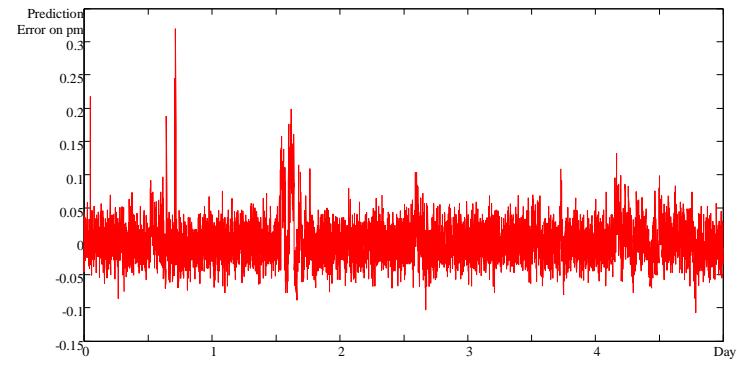

Figure 7: prediction error of $\mathrm{pm}$.

In order to illustrate this, the preceding models constructed with the data collected by airbox 1 are used with the data collected on airbox 2 .

The s-chart described part 3 is used in order to determine if a relearning is needed. The size of the samples is fixed to 240 minutes and so each sample contains 240 patterns. Figure 8 presents the s-chart obtained for the pm model used with data collected by airbox 2 .

This figure shows that, as awaited, the model is not accurate for the first sample of 240 data. So a relearning occurs on these 240 first data. This relearning allows to fit the model to these new condition and the model accuracy is satisfactory until the sample 25. For this sample, the value of the standard deviation is slightly outside the bounds and a second relearning is needed. So, for the 15 days of the experiments, only 2 relearning are needed to maintain a good accuracy of the model.

Figure 9 presents the prediction error for the pm pollutant. This figure shows that this strategy allows maintaining an accurate model even when the conditions change.

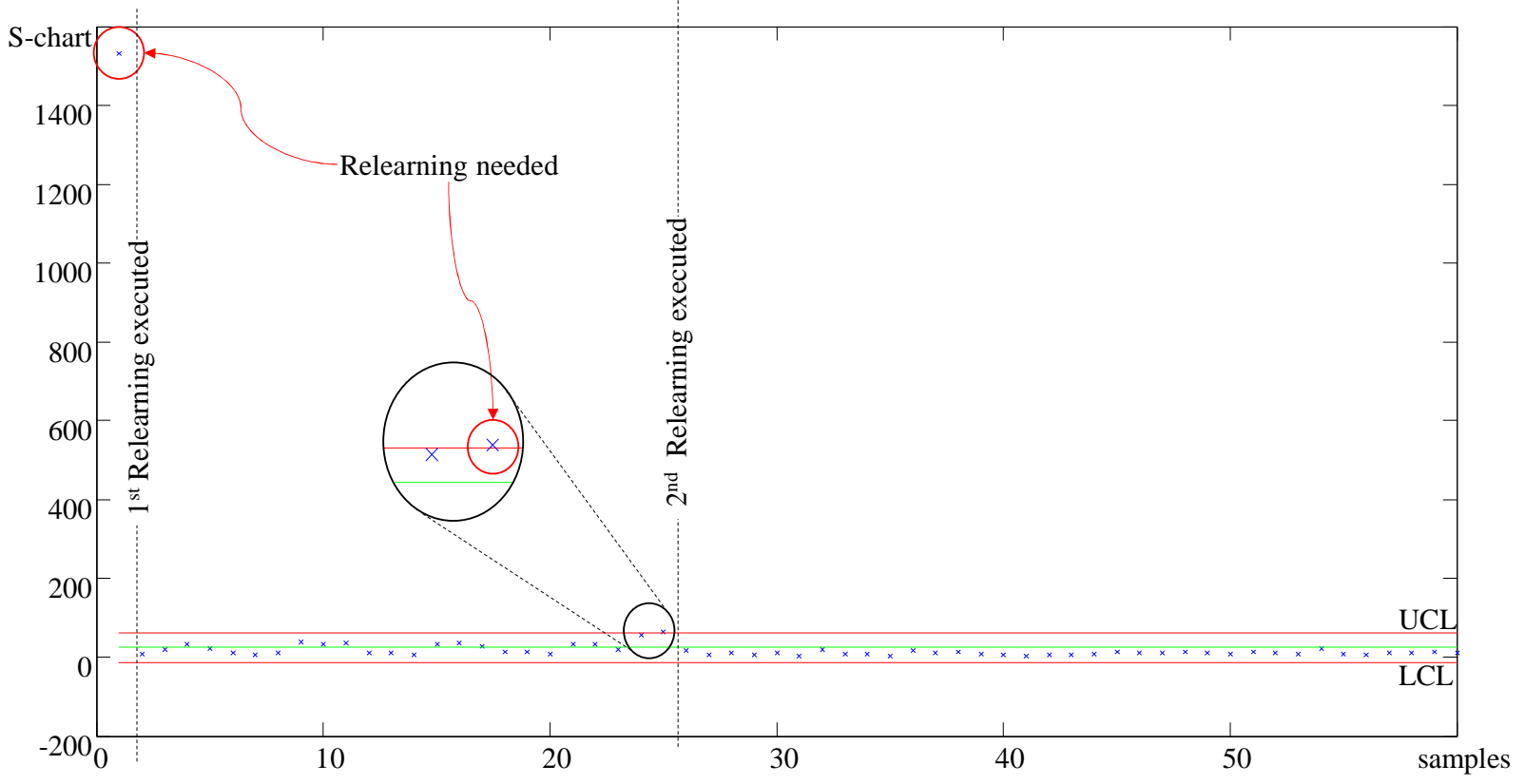

Figure 8: S-chart: monitoring of model accuracy. 


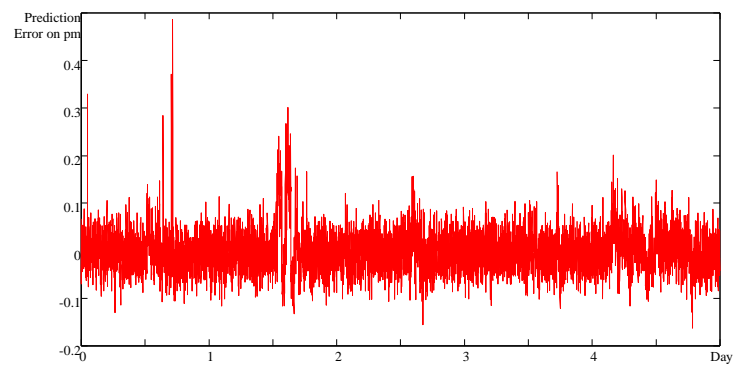

Figure 9: prediction error of pm for airbox 2.

\section{CONCLUSIONS}

This paper presents a new on-line monitoring strategy conserving the prediction model accuracy. This strategy is based on the use of a control chart to determine if a relearning is needed or not to adapt the model to an evolution of the reality. This approach is tested on a prediction model related to pollutants levels. The results show that neural networks are able to predict the evolution of pollutants. Moreover, the proposed monitoring strategy allows adapting quickly the considered model to new conditions and, in the same time, to limit the number of relearning needed. In future works, this approach will be compared with on-line learning on both accuracy and computational time.

\section{ACKNOWLEDGEMENTS}

The authors thanks the society Anaximen for their active financial and scientific support for this work.

\section{REFERENCES}

Alima, http://getalima.com, 2013.

Cybenko, G., 1989. Approximation by superposition of a sigmoidal function. Math. Control Systems Signals, 2, 4, 303-314.

Du, L., Ke, Y., Su, S., 2012. The Embedded Quality Control System of Product Manufacturing, Advanced Materials Research, 459, 510-513.

Engelbrecht, A.P., 2001. A new pruning heuristic based on variance analysis of sensitivity information. IEEE transactions on Neural Networks, 1386-1399

Funahashi, K., 1989. On the approximate realization of continuous mapping by neural networks. Neural Networks, 2, 183-192.
Han, H.G., Qiao, J.F., 2013. A structure optimisation algorithm for feedforward neural network construction. Neurocomputing, 99, 347-357.

Heskes, T., Wiegerinck, W., 1996. A theoretical comparison of batch-mode, online, cyclic, and almostcyclic learning. IEEE Transactions on Neural Networks, 7, 919-925.

Jones, A.P, 1999. Indoor air quality and health. Atmospheric Environment, 33, 28, 4535-4564.

Liang, N.Y., Huang, G.B., Saratchandran, P., Sundarajan, N., 2006. A fast and accurate online sequential learning algorithm for feedforward networks. IEEE Transactions on Neural Networks, 17, 1411-1423.

Ma, L., Khorasani, K., 2004. New training strategies for constructive neural networks with application to regression problems. Neural Network, 589-609.

Maroni, M., Seifert, B., Lindvall, T., 1995. Indoor Air Quality - a Comprehensive Reference Book. Elsevier, Amsterdam.

Nakama, T., 2009. Theoretical analysis of batch and online training for gradient descent learning in neural networks. Neurocomputing, 73, 151-159.

Nguyen, D., Widrow, B., 1990. Improving the learning speed of 2-layer neural networks by choosing initial values of the adaptative weights. Proc. of the Int. Joint Conference on Neural Networks IJCNN'90, 3, 21-26.

NIST/SEMATECH, 2012. e-Handbook of Statistical Methods, http://www.itl.nist.gov/div898/handbook/.

Setiono, R., Leow, W.K., 2000. Pruned neural networks for regression. $6^{\text {th }}$ Pacific RIM Int. Conf. on Artificial Intelligence PRICAI'O0, Melbourne, Australia, 500509

Shewhart, W.A., 1931. Economic Control of Quality of Manufactured Product, Van Nostrand Reinhold Company, Inc.: Princeton, NJ.

Spengler, J. D., Sexton, K., 1983. Indoor air pollution: a public health perspective. Science, 221, 9-17.

Tague, N.R., 2004. The Quality Toolbox, 2nd Edition, ASQ Quality Press, http://asq.org/learn-aboutquality/data-collection-analysistools/overview/control-chart.html.

Thomas, P., Bloch, G., 1997. Initialization of one hidden layer feedforward neural networks for non-linear system identification. 15 $5^{\text {th }}$ IMACS World Congress on Scientific Computation, Modelling and Applied Mathematics WC'97, 4, 295-300.

Thomas, P., Bloch, G., Sirou, F., Eustache, V., 1999. Neural modeling of an induction furnace using robust learning criteria. Journal of Integrated Computer Aided Engineering, 6, 1, 5-23.

Thomas, P., Suhner, M.C., Thomas, A., 2013. Variance Sensitivity Analysis of Parameters for Pruning of a Multilayer Perceptron: Application to a Sawmill Supply Chain Simulation Model. Advances in Artificial Neural Systems, Article ID 284570, http://dx.doi.org/10.1155/2013/284570

Walsh, P.J., Dudney, C.S., Copenhaver, E.D., 1987. Indoor air quality, ISBM 0-8493-5015-8. 